\title{
Performance of the SALVE-microscope: Atomic-resolution TEM Imaging at 20kV
}

Martin Linck $^{1}$, Peter Hartel ${ }^{1}$, Stephan Uhlemann ${ }^{1}$, Frank Kahl ${ }^{1}$, Heiko Müller ${ }^{1}$, Joachim Zach ${ }^{1}$, Johannes Biskupek $^{2}$, Marcel Niestadt ${ }^{3}$, Ute Kaiser ${ }^{2}$ and Max Haider ${ }^{1}$

1. Corrected Electron Optical Systems GmbH, Englerstr. 28, D-69126 Heidelberg, Germany

2. Central facility of electron microscopy, Ulm University, Albert-Einstein-Allee 11, D-89081 Ulm, Germany.

3. FEI Company, Achtseweg Noord 5, 5651 GG Eindhoven, Netherlands.

The SALVE project had been initiated to develop a dedicated low-voltage TEM that is corrected for both, spherical and chromatic aberration, in order to allow atomic resolution TEM observations on beam sensitive materials $[1,2]$.

The centerpiece of the SALVE III microscope is CEOS' new Cc-Cs-corrector that is based on the socalled Rose-Kuhn-Design [3,4]. The corrector is incorporated into a cubed FEI Titan Themis TEM and has been aligned for five accelerating voltages in the range from 20 to $80 \mathrm{kV}$.

During corrector design special care had to be taken to avoid the resolution limiting effects of thermal magnetic field noise (Johnson-Nyquist noise) that effectively limits the information transfer in Cc-corrected electron microscopes [5]. As seen in Table 1, these measures have been successful in that for all desired high-tensions the resulting final resolutions exceed the desired 50mrad aperture. Figure 1(a) exemplarily demonstrates the achieved information limit at an accelerating voltage of $20 \mathrm{kV}$. For the two perpendicular directions the Young's fringes on a purely amorphous 2nm tungsten sample significantly exceed the 50mrad radius. In order to "use" the transferred information adequately, the phase plate, i.e. the aberration function, has to be well-controlled beyond the 50mrad-angle. This requires access up to including 5th order axial aberrations, and -for a considerable field of view- control over off-axial aberrations. The aberration measurement in Figure 1(b) demonstrates that all unround axial aberrations as well as the off-axial aberrations can be tuned sufficiently small. At the same time, the round aberrations can be adjusted for a suitable phase contrast transfer function (indicated in green color). Consequently, as visible in Figure 1(c) atomic resolution imaging at 20kV becomes reality. Chromatic aberration causes inelastically scattered electrons, i.e. electrons of lower energy, to be focused much stronger. This is not true in the Cc-corrected instrument. Figure 1(d) compares the energydependent defocus effect at $20 \mathrm{kV}$ of a Cc-uncorrected TEM (red line, $\mathrm{Cc}=1.45 \mathrm{~mm}$ ) and the SALVE microscope (measurements: black dots, 3rd order fit: dashed line). The magnified area indicates that EFTEM with significant energy windows is possible even at $20 \mathrm{kV}$ enabling new modes of operation such as high-resolution EFTEM at very low voltages. [6]

\section{References:}

[1] U. Kaiser et al., Ultramicroscopy 111, Issue 8 (2011), 1239-1246.

[2] http://www.salve-project.de/

[3] H. Rose, Proc. 10th Eur. Congr. El. Micr. (Granada, Spain) (1992), 47.

[4] H. Rose, Patent Application DE 4204512 A 1 (1992).

[5] S. Uhlemann et al., Physical Review Letters 111(4) (2013), 046101.

[6] The authors acknowledge funding from the German Research Foundation (DFG) and the Ministry of Science, Research and the Arts (MWK) of the federal state Baden-Württemberg, Germany. 


\begin{tabular}{|c|c|c|c|}
\hline high voltage & wave length & 50 mrad resolution & SALVE results \\
\hline $20 \mathrm{kV}$ & $8.589 \mathrm{pm}$ & $171.8 \mathrm{pm}$ & $139 \mathrm{pm}$ \\
\hline $30 \mathrm{kV}$ & $6.979 \mathrm{pm}$ & $139.6 \mathrm{pm}$ & $115 \mathrm{pm}$ \\
\hline $40 \mathrm{kV}$ & $6.016 \mathrm{pm}$ & $120.3 \mathrm{pm}$ & $90 \mathrm{pm}$ \\
\hline $60 \mathrm{kV}$ & $4.866 \mathrm{pm}$ & $97.3 \mathrm{pm}$ & $83 \mathrm{pm}$ \\
\hline $80 \mathrm{kV}$ & $4.176 \mathrm{pm}$ & $83.5 \mathrm{pm}$ & $76 \mathrm{pm}$ \\
\hline
\end{tabular}

Table 1. Resulting information limit of the SALVE-microscope for different accelerating voltages. The resolution as determined from Young's fringes visibility on an amorphous tungsten sample significantly exceeds the $50 \mathrm{mrad}$ resolution for all high-tensions.
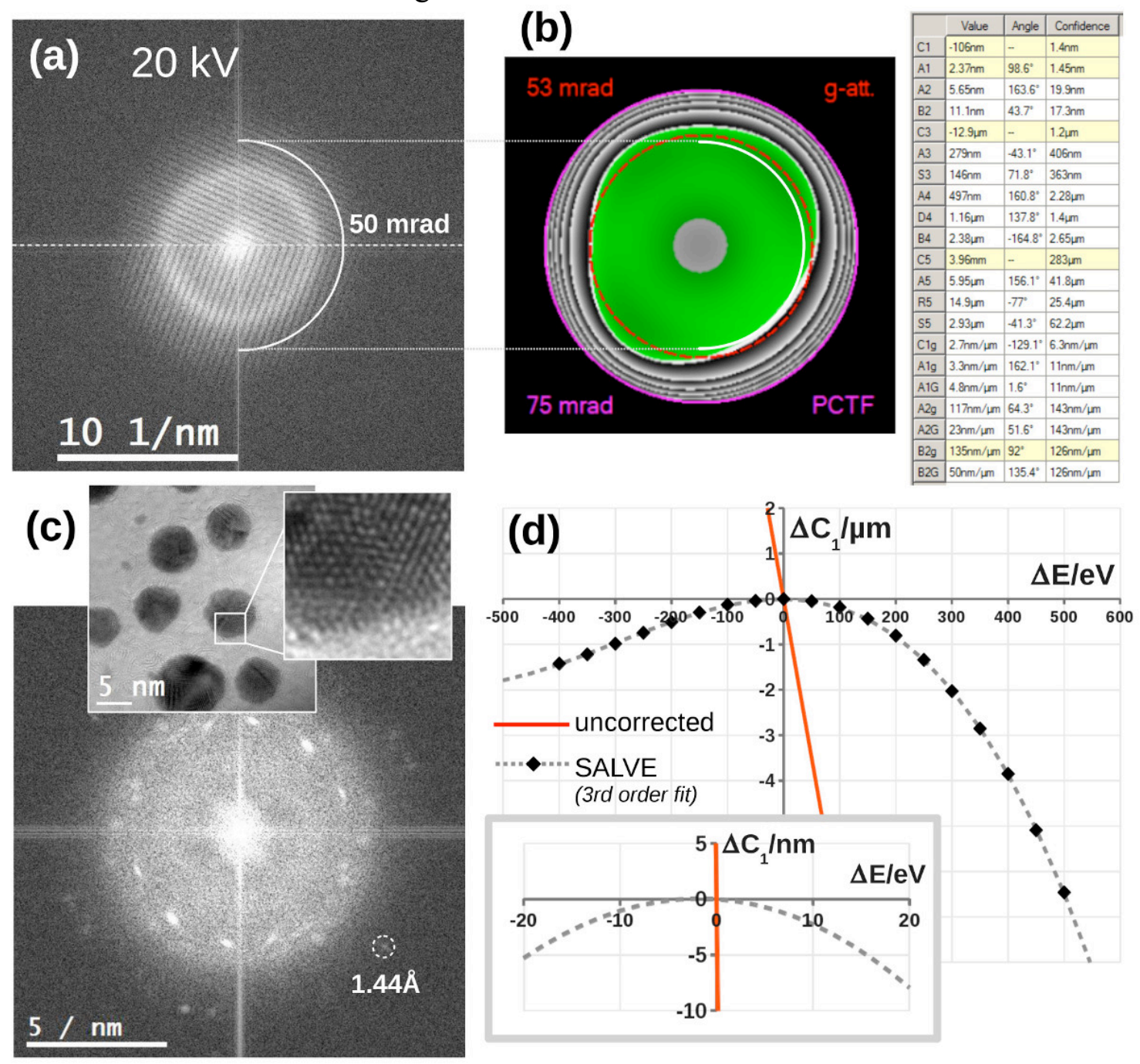

Figure 1. SALVE results at 20kV: (a) The Young's Fringes resolution test (in two directions) on an amorphous tungsten sample indicates an information limit beyond the 50mrad aperture. (b) At the same time, the wave aberration can be tuned up to in 5th order for uniform phase contrast beyond 50mrad (PCTF passband in green color, corresponding aberration coefficients tabulated). (c) Atomic resolution image of gold clusters at $20 \mathrm{kV}$. The 1.44 Angstrom reflections clearly show up in the corresponding FFT. (d) Defocus vs. energy offset at 20kV: Unlike the Cc-uncorrected TEM (red line) the SALVE corrector allows imaging without significant focus changes (dashed line). Within a $20 \mathrm{eV}$ window the defocus changes by only $2 \mathrm{~nm}$ due to the remaining higher order chromatic aberrations. 